\title{
Allo-parental care in Damaraland mole-rats is female biased and age dependent, though independent of testosterone levels
}

\section{Zöttl, Markus}

2018-09-01

Zöttl , M , Vullioud , P , Goddard , K, Torrents-Ticó , M, Gaynor , D , Bennett , N C \& Clutton-Brock , T 2018 , ' Allo-parental care in Damaraland mole-rats is female biased and age dependent, though independent of testosterone levels ', Physiology \& Behavior, vol. 193 , no. Part A , pp. 149-153 . https://doi.org/10.1016/j.physbeh.2018.03.021

http://hdl.handle.net/10138/311639

https://doi.org/10.1016/j.physbeh.2018.03.021

cc_by_nc_nd

acceptedVersion

Downloaded from Helda, University of Helsinki institutional repository.

This is an electronic reprint of the original article.

This reprint may differ from the original in pagination and typographic detail.

Please cite the original version. 


\section{Allo-parental care in Damaraland mole-rats is female biased and age dependent, though independent of testosterone levels}

Zöttl Markus ${ }^{1,5}$, Vullioud Philippe ${ }^{1}$, Goddard Katy ${ }^{3}$, Torrents-Ticó Miquel ${ }^{3,4}$, Gaynor David², Bennett C Nigel ${ }^{2}$ and Tim Clutton-Brock ${ }^{1,2}$

${ }^{1}$ Department of Zoology, University of Cambridge, Cambridge, UK

${ }^{2}$ Department of Zoology and Entomology, Mammal Research Institute, University of Pretoria, Pretoria, South Africa

${ }^{3}$ Kalahari Mole-rat Project, Kuruman River Reserve, Van Zylsrus, South Africa

${ }^{4}$ Metapopulation Research Centre (MRC), Department of Biosciences, University of Helsinki, Helsinki, Finland

${ }^{5}$ Ecology and Evolution in Microbial Model Systems, EEMiS, Department of Biology and Environmental Science, Linnaeus University, SE-391 82 Kalmar, Sweden

For: Physiology and Behavior - Special issue on non-maternal care

Correspondence: Markus Zöttl, mz338@cam.ac.uk

\section{Highlights}

- Allo-parental care in males and females is unaffected by experimental increases in testosterone levels.

- Allo-parental care decreases with the age of non-breeding helpers.

- Female breeders were more likely than female non-breeders to carry pups but male breeders were less likely to carry pups than male non-breeders, increasing the sex bias in parental care compared to allo-parental care. 


\begin{abstract}
In Damaraland mole-rats (Fukomys damarensis), non-breeding subordinates contribute to the care of offspring born to the breeding pair in their group by carrying and retrieving young to the nest. In social mole-rats and some cooperative breeders, dominant females show unusually high testosterone levels and it has been suggested that high testosterone levels may increase reproductive and aggressive behavior and reduce investment in allo-parental care, generating age and state-dependent variation in behavior. Here we show that, in Damaraland mole-rats, allo-parental care in males and females is unaffected by experimental increases in testosterone levels. Pup carrying decreases with age of the non-breeding helper while the change in social status from non-breeder to breeder has contrasting effects in the two sexes. Female breeders were more likely than female non-breeders to carry pups but male breeders were less likely to carry pups than male non-breeders, increasing the sex bias in parental care compared to allo-parental care. Our results indicate that testosterone is unlikely to be an important regulator of allo-parental care in mole-rats.
\end{abstract}

\title{
Keywords
}

Testosterone ; Allo-parental care ; Parental care ; Sex bias ; Age related polyethism 


\section{Introduction}

In cooperatively breeding vertebrates where non-breeders provide allo-parental care for offspring produced by dominant breeders (Cockburn 1998, Koenig and Dickinson 2016), the extent of investment in allo-parental care frequently changes with age of the helping individual (Heinsohn and Cockburn 1994, Clutton-Brock et al. 2000, Bruintjes and Taborsky 2011), differs between sexes (Heinsohn and Legge 1999, Clutton-Brock et al. 2002, CluttonBrock et al. 2003) and females reduce their investment in care behavior when achieving breeder status (Cant and Field 2001, Clutton-Brock et al. 2002, Heinsohn 2004). However, the physiological mechanisms regulating varying investment in allo-parental care are poorly understood.

It has been suggested that allo-parental and parental care are regulated by the same physiological mechanisms (Ziegler 2000, Tecot and Baden 2015, Schradin et al. 2017 this issue) and that testosterone may be a regulator of allo-parental and parental care (Wingfield et al. 1990, Ketterson and Nolan 1994, Trainor and Marler 2002, Schoech et al. 2004, Hau 2007, Schradin et al. 2017 this issue). In several cooperative birds and mammals, there is evidence that increases in testosterone levels are associated with increases in competition for breeding opportunities and reductions in allo-parental care. For example, in birds, periods with allo-parental care coincide with periods of low testosterone levels (Schoech et al. 1996, Vleck and Brown 1999, Pikus et al. 2017) while, in meerkats (Suricata suricatta), extraterritorial prospecting increases plasma testosterone in subordinates, who also show reduced investment in pup feeding after their return to the group (Young et al. 2005). There are also experimental results suggesting that in male prairie voles, (Microtus ochrogaster) testosterone applications during early development may suppress some forms of allo- 
parental care later in life (Roberts et al. 1996). However, experiments manipulating testosterone have rarely been conducted in cooperative breeders and it remains unclear whether increased testosterone reduces allo-parental care in these species.

In social mole-rats, non-breeders and breeders of both sexes provide direct care for the offspring, though non-breeding females may invest more in allo-parental care than males (Bennett and Faulkes 2000, Zöttl et al. 2016a). Group members carry young offspring to the safety of the communal nest and, in contrast to some other cooperatively breeding mammals, lactation is limited to a single breeding female in each group (Bennett and Jarvis 1988, Bennett 1990, Jarvis 1991, Lacey and Sherman 1991, Lacey and Sherman 1997). Pup carrying in mole-rats is displayed in a period after the offspring's birth when young wander out of the nest, or when mole-rat groups are disturbed and offspring are evacuated from an area of danger. As in some other cooperative breeders (Davies et al. 2016), testosterone increases when individuals transition from non-breeder to breeder (Clarke and Faulkes 1997, Lutermann et al. 2013) but its effects on parental and allo-parental care are unknown.

This study focuses on pup carrying since it is an obvious form of cooperative behavior (West et al. 2007) and because it is unlikely to yield immediate selfish or mutual benefits to caring individuals (Lukas and Clutton-Brock 2012, 2017). In laboratory groups of mole-rats, pup carrying can be elicited in a standardized behavioral test paradigm by placing pups outside the nest and observing which individuals carrying pups back to the nest. Our study uses a combination of observations and experiments on captive groups of Damaraland mole-rats (Fukomys damarenis) to investigate age- and status-dependent differences of pup carrying behavior and it tests the prediction that increased testosterone levels reduce the probability of pup carrying by non-breeding mole-rats. 


\section{Methods}

\section{Study animals and husbandry}

The animals used in this study were wild caught Damaraland mole-rats and their offspring (size range 2-19 individuals per group), bred and raised under captive conditions. The animals were captured around the Kuruman River Reserve in the Northern Cape in South Africa between February and September 2013, and subsequently housed in a rodent facility at the Kuruman River Reserve. The rodent facility consists of seven rooms which housed 54 breeding groups of mole-rats during the study period (typically 6-12 per room). All rooms were temperature controlled, allowing for different temperatures in winter and summer (Winter/Summer temperature $22 / 25$ Celsius) to reflect seasonal changes in their natural habitat. The mole-rats were either maintained in the group in which they had originally been captured or in new groups that were established by pairing one male and one female from different groups. All individuals were dye-marked to allow individual recognition and carried a passive implantable transponder for identification.

All groups were housed in artificial tunnel systems of 4 to 16 meters of PVC tunnels depending on group size. All tunnel systems included compartments for nesting and food storage, toilet areas and one large box. The upper halves of the tunnels had a transparent PET window enabling behavioral observation. Twice a day the mole-rats were fed ad libitum with sweet potatoes and cucumbers (i.e. we provided more food than a group would eat and removed food that was no longer fresh) and two to three vertical tunnels were refilled with clean sand to provide digging substrate. Once a day the tunnels were cleaned and confined toilet areas were rinsed with hot water. Occasionally apples, squash and potatoes were offered to enrich the diet. 


\section{Observational data collection}

Observational data were collected between $10^{\text {th }}$ November 2014 and $7^{\text {th }}$ February 2017 and the testosterone manipulation was conducted between $24^{\text {th }}$ of October 2015 and $2^{\text {nd }}$ of May 2016. We used a standardized behavioral assay within the group's tunnel system to record how likely individuals are to respond to young offspring by taking them between their teeth and carrying them to the nest, or another place in the tunnel system. We removed the entire litter from the nest and placed them in a standardized location in the tunnel system of the group. Subsequently, we recorded all interactions of the group members with the pups and recorded which individuals interacted with them, and which individuals carried pups to another place in the tunnel system or to the nest. All observations were conducted between 7 am and 11 am following an all-occurrence sampling protocol (Altmann 1974) on a handheld Android device operating the software Pocket Observer (Noldus, Wageningen). For each of the 127 litters, the behavioral assay was repeated on 3 different days between day 10 and day 15 after birth of every litter. At this age, pups have hair and show some mobility, but are still small enough to be carried back swiftly when they leave the nest.

\section{Testosterone manipulation experiment}

To experimentally increase testosterone levels and evaluate the consequences for alloparental care we developed a test paradigm that allowed us to test single individuals for their readiness to carry pups away from potentially dangerous situations. The experiment was conducted on 40 individuals ( 20 males, 20 females; Age range: 270 to 831 days, mean $=464$ days, $\mathrm{N}=33 ; \mathrm{N}=7$ individuals of unknown age $>831$ ) which were randomly chosen among laboratory reared or wild caught non-breeders above 75 grams from 12 different groups. Damaraland mole-rats in this age and weight range are likely to have undergone puberty and 
remain sexually suppressed until mating opportunities with unrelated individuals arise (Bennett et al. 1996).

We employed a within-subject test paradigm, where every test subject underwent both conditions, a control and an increased testosterone treatment, half of the subjects starting with the control and half with the treatment. In the testosterone treatment, the individuals were injected with an intramuscular injection of $0.1 \mathrm{mg}$ of testosterone propionate suspended in $0.1 \mathrm{ml}$ castor oil. In our study species, the injection of $0.1 \mathrm{mg}$ testosterone propionate has been shown to induce a 10 -fold increase of circulating plasma testosterone levels 24 hours post-injection (Vullioud 2017). A lower dose of $0.04 \mathrm{mg}$ testosterone propionate led to an increase in plasma testosterone levels on the day after injection but the increase was no longer significant after 48 hours in urine, whereas the higher dose of $0.1 \mathrm{mg}$ led to a significant increase in urinary testosterone (Vullioud 2017). As a control, we used an injection of $0.1 \mathrm{ml}$ pure castor oil.

Between 4 and $7 \mathrm{pm}$ on the first day of the experiment we anesthetized the test subject with vaporized isoflurane and injected the control or the treatment suspension intramuscularly in the hind leg. The allo-parental care test was conducted on the subsequent day between 7 and $12 \mathrm{am}$. We repeated the same procedure one week later with the reverse treatment.

The allo-parental care test was conducted in a white plastic box $(60 \mathrm{~cm} \times 40 \mathrm{~cm} \times 30 \mathrm{~cm})$ which was placed beside the tunnel system of the test subjects. The bottom of the box was covered with a thin layer of sand. First, we removed a stimulus pup from the group's tunnel system and placed it in the corner of the test box. Subsequently, we removed the test subject from the tunnel system and placed it beside the pup in the corner of the box. Immediately after the test subject entered the test box, we started a focal observation on the test subject and recorded all pup carrying activity during the next 300 seconds. The test subject was always 
tested with the same stimulus pup, from their own group, in both the control and the testosterone treatment. The experiments were conducted when the stimulus pups were in a body mass range between 25 and 35 grams. When reaching this stage of development, the stimulus pups are already independently leaving the nest but are occasionally carried back to the nest in case of disturbance or threats. Pups reach this stage earliest on day 20 after birth (mean age at 25 gram = 40 days). The set-up of our allo-parental care test resulted in an overall mean duration of pup carrying of 90.4 seconds with a range from 0 seconds to 291 seconds.

\section{Statistical analysis}

Since we focused on the probability of providing allo-parental or parental care, our observations were designed to record whether or not an individual provided care (i.e. carried a pup) when encountering a situation where care behavior may be displayed. During our observational data collection in the tunnel systems, we recorded for each individual of the group how often it encountered a pup outside the nest and whether or not it responded by carrying the pup back to the nest or to another place in the tunnel system. Subsequently, for all individuals that encountered a pup at least once in an observation period, we calculated the proportion of encounters that involved pup carrying (number of encounters that led to the pup being carried/ total number of encounters). Those that did not encounter a pup, and thus did not have the opportunity to express offspring care, were excluded from subsequent analysis. We included all non-reproductive individuals that were heavier than $50 \mathrm{~g}$ which resulted in an age cohort of non-breeding individuals ranging from 113 to 1194 ( mean $=440$ ) days of age. The breeders with known age ranged from 538 to 1194 (mean $=845$ ) days of age. In total our dataset included 391 individuals of which 195 were female and 196 were male. 
To analyze how likely non-breeding individuals are to carry pups and whether this is related to age and sex, we fitted a generalized linear mixed model assuming binomial error structure (GLMM, logit link function) with the proportion of successful carries as the response variable and the age (in days), the sex of the individuals and their interaction as predictors. To control for non-independence of observations due to repeated sampling we added the individual identity, the litter identity and the group identity as independent random factors. To evaluate whether the pup care probability shows a similar ontogeny as suggested for other cooperative behaviors in the species (cf. Zöttl et al. 2016a), we additionally specified the same model but restricted the data to all non-breeders up to 600 days of age. In both models, the age covariate was z-transformed.

To evaluate how non-breeder and breeding individuals differ in the probability of providing care for offspring in the group, we fitted a GLMM assuming binomial error structure with the proportion of successful carries as the response variable and the breeding status (breeder/non-breeder), the sex of the individual and their interaction as predictors. Breeder females are morphologically distinguishable and in males, we assigned breeder status to the socially dominant individual by the direction of pairwise submission behavior. Restricting the data set to known breeder males (due to group establishment in the laboratory) did not change the results qualitatively.

To test whether experimental increases in testosterone affect pup carrying, we fitted a linear mixed effect model with the duration the stimulus pup was carried during the experiment as response and the condition (Control/ Treatment), sex and their interaction as predictors. We also fitted the individual identity as random effect. P-values presented for this model were calculated using the log likelihood ratio test under stepwise model simplification. 
The residuals of the models were visually inspected for independence and normality, and the overdispersion factor (residual scaled deviance / the residual degrees of freedom) of GLMMs presented in this paper did not indicate overdispersion (0.68-0.83). All models presented were estimated using the software R version 3.3.2 and the package Ime4 (Bates et al. 2011).

\section{Ethical note}

The laboratory facility at the Kuruman River Reserve is approved by the NSPCA South Africa and all studies are subject to approval by the ethics committee of the University of Pretoria (Permits EC-089-12, EC-009-13 and SOP-004-13).

\section{Results}

In non-breeding mole-rats, pup carrying was sex biased towards females and decreased with the helper's age (Figure $1 \mathrm{a}$ and $b$, GLMM; Sex: Estimate $=-0.61 \pm 0.14, p<0.001$, Age: Estimate $=-0.22 \pm 0.06, \mathrm{p}<0.001, \mathrm{~N}=1069$ observations, $\mathrm{N}=257$ individuals, $\mathrm{N}=47$ groups). Nonbreeding males and females showed similar reductions in pup carrying with increasing age (GLMM; Sex $\times$ Age: Estimate $=0.026 \pm 0.11, p=0.81, N=1069$ observations, $N=257$ individuals, $\mathrm{N}=47$ groups). In a more limited data set up to the age of 600 days, we did not detect significant changes in pup carrying in males and females (GLMM; Sex: Estimate $=-0.70 \pm 0.16$, $p<0.001$, Age: Estimate $=0.06 \pm 0.08, p=0.42$, Sex $x$ Age: Estimate $=-0.18 \pm 0.11, p=0.09, N=885$ observations, $\mathrm{N}=247$ individuals, $\mathrm{N}=44$ groups). 

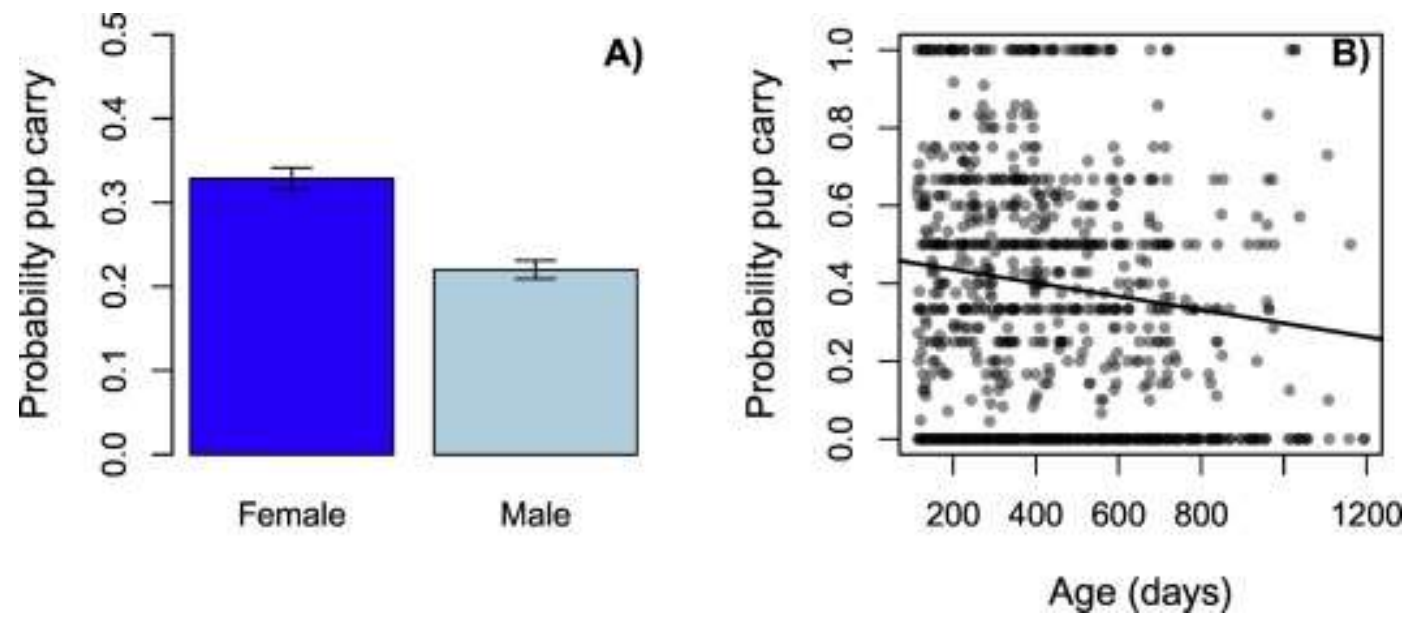

Figure 1. Probability of pup carrying in non-breeding Damaraland mole-rats. (A) Sex differences. Bars represent mean standard error. (B) Plotted against the age (in days) of the individual. Data points represent the raw data and the trend line is the prediction from the equivalent linear model. Sample sizes are $\mathrm{N}=1069$ observations, $\mathbf{N}=\mathbf{2 5 7}$ individuals, $\mathrm{N}=\mathbf{4 7}$ groups. See main text for statistics.

Breeder and non-breeder males and females showed a different pattern. While pup carrying was more frequently observed in breeding females than in non-breeding females, it was less frequently observed in breeding males than in non-breeding males, with the result that sex differences in allo-parental care were smaller than those in parental care (Figure 2; GLMM; Sex: Estimate $=-0.55 \pm 0.13, p<0.001$, Status: Estimate $=0.27 \pm 0.15, p=0.07$, Sex $x$ Status: Estimate $=-0.81 \pm 0.25, \mathrm{p}<0.0001, \mathrm{~N}=1849$ observations, $\mathrm{N}=391$ individuals, $\mathrm{N}=53$ groups).

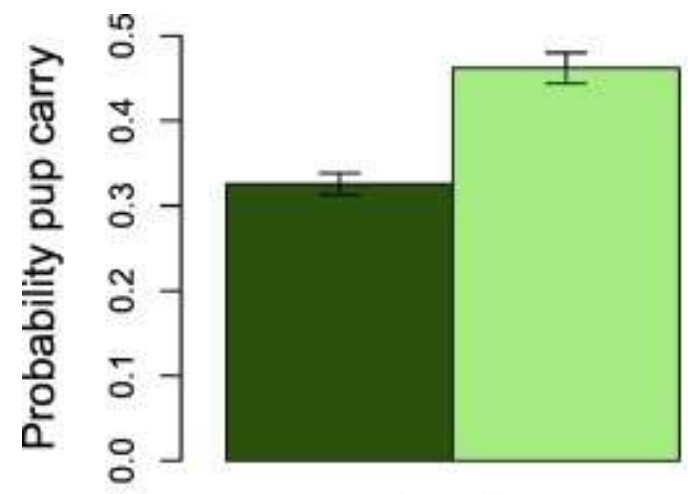

Female

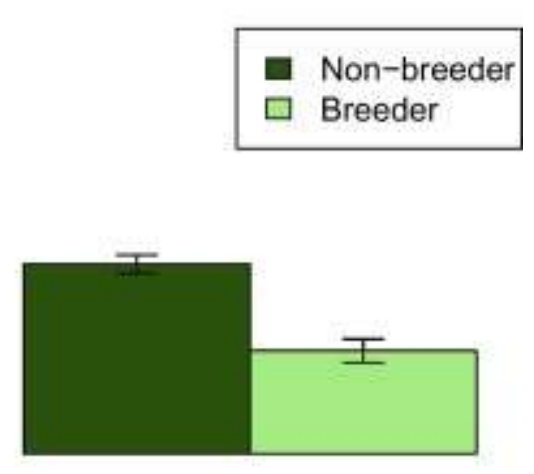

Male

Figure 2. Probability of pup carrying for female and male mole-rats by breeding status. Bars represent mean standard error. Sample sizes are $\mathrm{N}=1812$ observations, $\mathrm{N}=\mathbf{3 8 2}$ individuals, $\mathrm{N}=53$ groups. See main text for statistics. 
The duration individuals carried pups during the test trials did not differ between the testosterone and the control treatment (Figure 3; Treatment: Estimate $=-5.4 \pm 12.7, p=0.67$ ). Also, there was no indication of sex specific responses to the testosterone treatment (Sex: Estimate $=-26.23 \pm 24.1, p=0.26$, Treatment $x$ Sex: Estimate $=10.73 \pm 25.9, p=0.67)$.

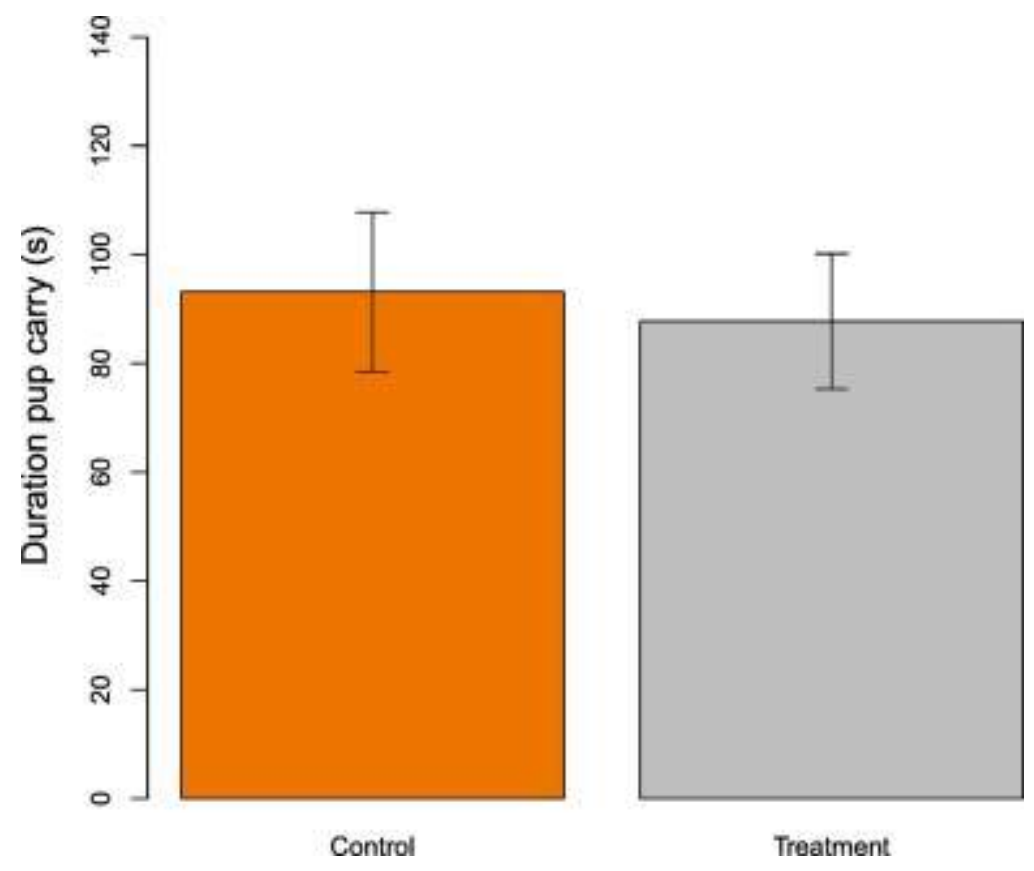

Figure 3 Duration of pup carrying (in seconds) provided per observation of $\mathbf{3 0 0}$ seconds for non-breeding Damaraland mole-rats after an experimental increase in testosterone (Treatment) and after a vehicle only control (Control). $\mathrm{N}=\mathbf{4 0}$ individuals. Bars represent mean and standard error. See main text for statistics.

\section{Discussion}

Our study suggests that allo-parental care decreases with the age of the non-breeding helper, that the change in social status from non-breeder to breeder has contrasting effects for pup care in the two sexes and that allo-parental care in male and female Damaraland mole-rats may be independent of testosterone levels. We focused on one aspect of direct care, carrying pups back to the safety of the nest because non-breeding individuals display a behavior that provides benefits to the breeder's offspring without yielding immediate benefits to the acting non-breeder. In contrast, some important cooperative behaviors such as cooperative 
foraging, territory defense or huddling for thermoregulation have immediate benefits to the acting non-breeder and may be explained by mutualistic benefits to all group members or as a selfish behavior that provides benefits to the offspring as a by-product (West et al. 2007). Pup carrying in mole-rats is energetically cheaper than some forms of allo-parental care in other cooperatively breeding mammals and birds, and some other cooperative behaviors in mole-rats. In meerkats, babysitters often forego foraging for an entire day and lose on average $5 \%$ of their body mass during this time period (Young et al. 2006, Clutton-Brock and Manser 2016) and in cooperatively breeding birds, chick feeding reduces the food intake of helpers over a long period (Cockburn 1998). In mole-rats, non-breeders may engage in energetically costly cooperative foraging, amounting to $7-10 \%$ of their time budget, by digging a large underground tunnel system that provides food and safety for the entire group (Faulkes and Bennett 2016, Zöttl et al. 2016a). In contrast, direct allo-parental care in mole-rats is short-lasting and may only be provided in rare events of danger in the tunnel system or when a nest needs to be evacuated. Although the fitness benefits of carrying pups back to the nest have never been quantified, they may be high in cases where the mole-rat group is threatened by a predator.

Our results suggest that short-lasting, peak levels of testosterone do not affect pup carrying in Damaraland mole-rats. Some previous studies have produced results that are consistent with the suggestion that high testosterone levels may reduce allo-parental care and it has been proposed that testosterone may be the proximate mechanism underpinning female biased, and age-dependent allo-parental care in cooperatively breeding birds and mammals (Schoech et al. 1996, Vleck and Brown 1999, Young et al. 2005, Pikus et al. 2017). However, another experimental study also showed no effect of experimentally increased testosterone on allo-parental care (Raynaud and Schradin 2014) and a meta-analysis in this special issue 
suggests that mechanisms underpinning allo-parental care and parental care may often differ (Schradin et al. 2017 this issue). In social mole-rats, breeding females that usually have the highest levels of testosterone (Clarke and Faulkes 1997, Lutermann et al. 2013) also show the highest responsiveness to pups in need (this study). Together, these studies suggest that high testosterone may be the consequence of competitive social interaction and group instability and that selection may favor offspring care to remain independent from testosterone regulation. This may allow individuals that are exposed to intra-sexual competition to show increased testosterone levels without compromising care for offspring (Lynn et al 2005, Clutton-Brock et al. 2006, Aubin-Horth et al. 2007) which would be particularly important in species with year-round breeding, like Damaraland mole-rats (Bennett and Jarvis 1988, Jarvis and Bennett 1993), where mating activities and periods with offspring care frequently overlap.

In Damaraland mole-rats, older non-breeding individuals were less likely to carry young offspring back to the safety of the nest than younger individuals, suggesting that allo-parental care in mole-rats declines with increasing chances of independent reproduction. This resembles patterns found in some other social vertebrates such as cooperatively breeding meerkats (Clutton-Brock et al. 2003, Young et al. 2005), cooperatively breeding fish (Bergmuller et al. 2005, Zöttl et al. 2013a, Zöttl et al. 2013b), female prairie voles (Lonstein and De Vries 2000, 2001) and Californian mice (Gubernick and Laskin 1994).

Age-related changes in pup carrying differ from similar changes in cooperative burrowing. Whereas the development of investment in cooperative burrowing is characterized by a steady increase of investment during the first year of life (Zöttl et al. 2016a), our data do not provide evidence for a similar, early-life surge in pup carrying. In the second year of their life, when the first individuals reach their adult body mass and dispersal-age (Zöttl et al. 2016b, 
Torrents-Tico et al. 2018), investment in cooperative burrowing and pup carrying declines, which may coincide with dispersal from the group (Zöttl et al. 2016a). The reason for the contrasting patterns of allo-parental care and cooperative foraging early in life may be the different energetic demand of these behaviors. Whereas carrying a pup back to the safety of the nest lasts only some seconds to minutes and is energetically cheap, cooperative burrowing entails substantial costs that younger, growing individuals may need to avoid.

Our study suggests that variation in pup carrying in Damaraland mole-rats is largely a consequence of sex, age and social status. Although testosterone has been shown to co-vary with some of these traits (Clarke and Faulkes 1997, Lutermann et al. 2013), our results indicate that pup carrying is independent of increased testosterone in non-breeding individuals. Our study raises the question whether testosterone may affect pup carrying behavior through long-lasting developmental effects, or whether other hormones such as corticosterone and prolactin may have more profound effects on pup carrying in mole-rats.

\section{Acknowledgements}

We thank Rute Mendonca, Adam Mitchell, Andrea Kraus, Niklas Paulsson, Kyle Finn, Sally Bornbusch, Sean McGregor, Laura Higgs, Rachel Kwok, Francesco Santi, Natasha Waite, Holly English, Monica Fondseca, and Alexandra Jebb for help with maintaining the study population and data collection. Many thanks to Jack Thorley for comments on an earlier draft of this manuscript. Tim Vink and lan Stevenson provided invaluable assistance with data management and logistic support, and Christina Kooper, JP Netshanger, Steven Koper and Dawid Van Wyk were a great help in taking care of our study animals. We thank the Kalahari Research Trust for access to the research facilities and Marta Manser and the University of Zurich for their contribution to maintaining the Kalahari Research Centre. This study was 
funded by a European Research Council grant to TCB (294494) and a Jacobs foundation grant

to MZ. We are grateful to the Northern Cape Department of Environment and Nature

Conservation for permission to conduct research in the Northern Cape.

\section{References}

Altmann, J. 1974. Observational Study of Behavior: Sampling Methods. Behavior 49:227-265. Aubin-Horth, N., J. K. Desjardins, Y. M. Martei, S. Balshine, and H. A. Hofmann. 2007. Masculinized dominant females in a cooperatively breeding species. Molecular Ecology 16:1349-1358.

Bates, D., M. Maechler, and B. Bollinge. 2011. Ime4: Linear mixed-effects models using S4 classes.

Bennett, N. 1990. Behaviour and social organization in a colony of the Damaraland mole-rat Cryptomys damarensis. Journal of Zoology 220:225-247.

Bennett, N., C. Faulkes, and A. Molteno. 1996. Reproductive suppression in subordinate, nonbreeding female Damaraland mole-rats: two components to a lifetime of socially induced infertility. Proceedings of the Royal Society of London B: Biological Sciences 263:1599-1603.

Bennett, N. C., and C. G. Faulkes. 2000. African mole-rats: ecology and eusociality. Cambridge University Press.

Bennett, N. C., and J. U. Jarvis. 1988. The social structure and reproductive biology of colonies of the mole-rat, Cryptomys damarensis (Rodentia, Bathyergidae). Journal of Mammalogy:293-302.

Bergmuller, R., D. Heg, and M. Taborsky. 2005. Helpers in a cooperatively breeding cichlid stay and pay or disperse and breed, depending on ecological constraints. Proceedings of the Royal Society B-Biological Sciences 272:325-331.

Bruintjes, R., and M. Taborsky. 2011. Size-dependent task specialization in a cooperative cichlid in response to experimental variation of demand. Animal Behaviour 81:387394.

Cant, M. A., and J. Field. 2001. Helping effort and future fitness in cooperative animal societies. Proceedings of the Royal Society of London B: Biological Sciences 268:19591964.

Clarke, F., and C. Faulkes. 1997. Dominance and queen succession in captive colonies of the eusocial naked mole-rat, Heterocephalus glaber. Proceedings of the Royal Society of London B: Biological Sciences 264:993-1000.

Clutton-Brock, T., and M. Manser. 2016. Meerkats: cooperative breeding in the Kalahari. Cooperative breeding in vertebrates (eds WD Koenig, JL Dickinson):294-317.

Clutton-Brock, T. H., P. N. M. Brotherton, M. J. O'Riain, A. S. Griffin, D. Gaynor, L. Sharpe, R. Kansky, M. B. Manser, and G. M. Mcllrath. 2000. Individual contributions to babysitting in a cooperative mongoose, Suricata suricatta. Proceedings of the Royal Society of London Series B-Biological Sciences 267:301-305. 
Clutton-Brock, T. H., S. J. Hodge, G. Spong, A. F. Russell, N. R. Jordan, N. C. Bennett, L. L. Sharpe, and M. B. Manser. 2006. Intrasexual competition and sexual selection in cooperative mammals. Nature 444:1065-1068.

Clutton-Brock, T. H., A. F. Russell, and L. L. Sharpe. 2003. Meerkat helpers do not specialize in particular activities. Animal Behaviour 66:531-540.

Clutton-Brock, T. H., A. F. Russell, L. L. Sharpe, A. J. Young, Z. Balmforth, and G. M. Mcllrath. 2002. Evolution and development of sex differences in cooperative behavior in meerkats. Science 297:253-256.

Cockburn, A. 1998. Evolution of helping behavior in cooperatively breeding birds. Annual Review of Ecology and Systematics 29:141-177.

Davies, C. S., K. N. Smyth, L. K. Greene, D. A. Walsh, J. Mitchell, T. Clutton-Brock, and C. M. Drea. 2016. Exceptional endocrine profiles characterise the meerkat: sex, status, and reproductive patterns. Scientific Reports 6.

Faulkes, C. G., and N. C. Bennett. 2016. Damaraland and naked mole-rats: Convergence of social evolution. Cooperative Breeding in Vertebrates: Studies of Ecology, Evolution, and Behavior:338.

Gubernick, D. J., and B. Laskin. 1994. Mechanisms influencing sibling care in the monogamous biparental California mouse, Peromyscus californicus. Animal Behaviour 48:12351237.

Hau, M. 2007. Regulation of male traits by testosterone: implications for the evolution of vertebrate life histories. BioEssays 29:133-144.

Heinsohn, R., and A. Cockburn. 1994. Helping Is Costly to Young Birds in Cooperatively Breeding White-Winged Choughs. Proceedings of the Royal Society of London Series B-Biological Sciences 256:293-298.

Heinsohn, R., and S. Legge. 1999. The costs of helping. Trends Ecol.Evol. 14:53-57.

Heinsohn, R. G. 2004. Parental care, load-lightening, and costs. Ecology and evolution of cooperative breeding in birds. Cambridge University Press, Cambridge:67-80.

Jarvis, J., and N. Bennett. 1993. Eusociality has evolved independently in two genera of bathyergid mole-rats-but occurs in no other subterranean mammal. Behavioral Ecology and Sociobiology 33:253-260.

Jarvis, J. U. M. 1991. Reproduction of Naked Mole-Rats. Pages 384-425 in P. W. Sherman, J. U. M. Jarvis, and R. D. Alexander, editors. The biology of the naked mole-rat. Princ. Univ. Press, Princeton, N.J.

Ketterson, E. D., and V. Nolan. 1994. Male parental behavior in birds. Annual Review of Ecology and Systematics 25:601-628.

Koenig, W. D., and J. L. Dickinson. 2016. Cooperative breeding in vertebrates: studies of ecology, evolution, and behavior. Cambridge University Press.

Lacey, E. A., and P. W. Sherman. 1991. Social organization of Naked Mole-Rat colonies: evidence for divisions of labor. Pages 275-336 in P. W. Sherman, J. U. M. Jarvis, and R. D. Alexander, editors. The biology of the naked mole-rat. Princeton Univ. Press, Princeton N. J.

Lacey, E. A., and P. W. Sherman. 1997. Cooperative breeding in naked mole-rats. Pages 267301 in N. G. Solomon and J. A. French, editors. Cooperative Breeding in Mammals. Cambridge University Press, Cambridge.

Lonstein, J. S., and G. J. De Vries. 2000. Influence of gonadal hormones on the development of parental behavior in adult virgin prairie voles (Microtus ochrogaster). Behavioural brain research 114:79-87. 
Lonstein, J. S., and G. J. De Vries. 2001. Social influences on parental and nonparental responses toward pups in virgin female prairie voles (Microtus ochrogaster). Journal of Comparative Psychology 115:53.

Lukas, D., and T. Clutton-Brock. 2012. Cooperative breeding and monogamy in mammalian societies. Proceedings of the Royal Society of London B: Biological Sciences:rspb20112468.

Lukas, D., and T. Clutton-Brock. 2017. Climate and the distribution of cooperative breeding in mammals. Open Science 4:160897.

Lutermann, H., A. J. Young, and N. C. Bennett. 2013. Reproductive status and testosterone among females in cooperative mole-rat societies. General and Comparative Endocrinology 187:60-65.

Lynn, S. E., B. G. Walker \& J. C. Wingfield, J. C. 2005. A phylogenetically controlled test of hypotheses for behavioral insensitivity to testosterone in birds. Hormones and Behavior, 47: 170-177.

Pikus, A. E., S. Guindre-Parker, and D. R. Rubenstein. 2017. Testosterone, social status and parental care in a cooperatively breeding bird. Hormones and Behavior.

Raynaud, J., and C. Schradin. 2014. Experimental increase of testosterone increases boldness and decreases anxiety in male African striped mouse helpers. Physiology \& Behavior 129:57-63.

Roberts, R. L., A. Zullo, E. A. Gustafson, and C. S. Carter. 1996. Perinatal steroid treatments alter alloparental and affiliative behavior in prairie voles. Hormones and Behavior 30:576-582.

Schoech, S., J. Raynolds, and R. Boughton. 2004. Endocrinology.in K. WD and J. Dickinson, editors. Ecology and evolution of cooperative breeding in birds. Cambridge University Press, Cambridge.

Schoech, S. J., R. L. Mumme, and J. C. Wingfield. 1996. Delayed breeding in the cooperatively breeding Florida scrub-jay (Aphelocoma coerulescens): inhibition or the absence of stimulation? Behavioral Ecology and Sociobiology 39:77-90.

Schradin, C., P. Vuarin, and R. Rimbach. 2017. The neoteny-helper hypothesis: When to expect and when not to expect endocrine mechanisms to regulate allo-parental care? Physiology \& Behavior, this issue.

Tecot, S., and A. L. Baden. 2015. Primate allomaternal care. Emerging Trends in the Social and Behavioral Sciences: An Interdisciplinary, Searchable, and Linkable Resource.

Torrents-Tico, M., N. C. Bennett, J. U. Jarvis, and M. Zöttl. 2018. Early growth affects dispersal success in social mole rats - but not the duration of philopatry. Biology letters, 14 (2), 20180005

Trainor, B. C., and C. A. Marler. 2002. Testosterone promotes paternal behaviour in a monogamous mammal via conversion to oestrogen. Proceedings of the Royal Society of London B: Biological Sciences 269:823-829.

Vleck, C. M., and J. L. Brown. 1999. Testosterone and social and reproductive behaviour in Aphelocoma jays. Animal Behaviour 58:943-951.

Vullioud, P. 2017. Thesis Chapter 5: Effects of testosterone on cooperative and social behaviour in subordinate females. University of Cambridge, Cambridge.

West, S. A., A. S. Griffin, and A. Gardner. 2007. Social semantics: altruism, cooperation, mutualism, strong reciprocity and group selection. Journal of Evolutionary Biology 20:415-432. 
Wingfield, J. C., R. E. Hegner, A. M. Dufty Jr, and G. F. Ball. 1990. The" challenge hypothesis": theoretical implications for patterns of testosterone secretion, mating systems, and breeding strategies. The American Naturalist 136:829-846.

Young, A. J., A. A. Carlson, and T. Clutton-Brock. 2005. Trade-offs between extraterritorial prospecting and helping in a cooperative mammal. Animal Behaviour 70:829-837.

Young, A. J., A. A. Carlson, S. L. Monfort, A. F. Russell, N. C. Bennett, and T. Clutton-Brock. 2006. Stress and the suppression of subordinate reproduction in cooperatively breeding meerkats. Proceedings of the National Academy of Sciences of the United States of America 103:12005-12010.

Ziegler, T. E. 2000. Hormones associated with non-maternal infant care: a review of mammalian and avian studies. Folia Primatologica 71:6-21.

Zöttl, M., L. Chapuis, M. Freiburghaus, and M. Taborsky. 2013a. Strategic reduction of help before dispersal in a cooperative breeder. Biology Letters 9.

Zöttl, M., D. Heg, N. Chervet, and M. Taborsky. 2013b. Kinship lowers alloparental care where helpers pay-to-stay. Nature Communications 4:1341.

Zöttl, M., P. Vullioud, R. Mendonça, M. T. Ticó, D. Gaynor, A. Mitchell, and T. Clutton-Brock. 2016a. Differences in cooperative behavior among Damaraland mole rats are consequences of an age-related polyethism. Proceedings of the National Academy of Sciences 113:10382-10387.

Zöttl, M., J. Thorley, D. Gaynor, N. C. Bennett, and T. Clutton-Brock. 2016b. Variation in growth of Damaraland mole-rats is explained by competition rather than by functional specialization for different tasks. Biology Letters 12. 